\title{
USING AUTONOMOUS MODULAR MATERIAL HANDLING EQUIPMENT FOR MANUFACTURING FLEXIBILITY
}

\section{Björn Johansson}

Department of Product and Production Development

Chalmers University of Technology

Gothenburg, 412 96, SWEDEN
Edward J. Williams

Production Modeling Corporation

Three Parklane Boulevard, Suite 1006 West

Dearborn, MI 48126, U.S.A.

Tord Alenljung

Department of Signals and Systems

Chalmers University of Technology

Gothenburg, 412 96, SWEDEN

\begin{abstract}
This paper describes a modular autonomous material handling equipment solution for flexible automation. Discrete Event Simulation is in this case used as a tool for shortening time spent in many different phases of a manufacturing systems lifecycle. The paper presents the concept of autonomous modular material handling equipment, and how simulation is used as a support tool and lead time reducer in each lifecycle phase. Furthermore, we describe the knowledge levels needed for using the simulation support and conclude with examples of how this methodology are reducing lead times within a company.
\end{abstract}

\section{INTRODUCTION}

The demands on manufacturing industry are ever changing, and become more and more challenging (Nahmias 2001). Fulfilling the customer requirements is not good enough. Products have to be produced before customer needs in order to be first on the market. Needs can be created through commercials and other enticements. Meanwhile the lifecycles of products and production systems become shorter and shorter and the low-cost countries, with labor flexibility as the main tool for production planning and control, are gaining more and more industrial competitiveness. This is a threat in itself to European SME's, since today's inflexible automated manufacturing equipment has a hard time competing with the labor flexibility used in low cost countries. The above statements put very high pressure on the future production facilities in the western world. The need for truly flexible manufacturing systems which can support many products and product variants, is vital to the maintenance of competitiveness. There are many automation solutions available on the market today; however, their potential is not fully utilized. A chain is no stronger than its weakest link and the chain in automated production facilities is long. The chain consists of not only the equipment; but also the usage of the equipment in terms of working procedures, organizational structure, knowledge and learning environments for continuous improvements and the striving to keep the competencies within the company. Additionally, automation equipment sets demands on process efficiency, including communication, PDM, ERP, Production IT in combination with the empowerment of operators which is one of the key enablers for successful manufacturing in the factory of the future.

In order to empower operators in a reconfigurable modular manufacturing system, the necessity of well developed Human Machine Interfaces (HMI) is of the greatest importance (Wickens et al. 2004). One successful prototype plant has been made in the concept of PLM (Product and Process Lifecycle Management), which is proof of the concept, and a taste of future automation solutions for modern countries.

Large companies who sell automation software solutions, like ERP and PDM, often take the upper hand over SME who invest in their solutions. There is a need for a complete reconfigurable modular manufacturing system which can be easily adapted to product changes and demands. This system will also need to have a built-in software interface, including extensive HMI's for operators, which give the operators more control and responsibility. This increased work content will keep the production facilities competitive and profitable in the western world, caused by the demand of competence levels on the em- 
ployees. The approach of an intelligent modular reconfigurable manufacturing system will last through many products and product variants. It will also support SME in the competitive environment on the future market, since the long term investment cost will be much lower with modular autonomous material handling equipment. This paper will examine the possibilities concerning autonomous modular material handling equipment for manufacturing flexibility.

\section{AUTONOMOUS MODULAR MATERIAL HANDLING EQUIPMENT}

There are numerous institutes and research departments looking into the area of intelligent manufacturing systems, in the context of core machine and/or process intelligence (Koren et al. 1999, Koren et al. 2001, Mehrabi and Ulsoy 1997). Most of these approaches focus on the core processes in each company, for example grinding machines, milling machines, multi-task machines etc., plus how to reconfigure and control them in order to support high productivity. Many of these approaches ignore the material handling aspects of the manufacturing system entirely. However, the approach presented in this paper represents another point of view. We try to control all activities by looking at the manufacturing system with the material handling equipment as the driving force. Supervision, flow control, and dynamic buffering capabilities are some examples of effects generated by using intelligent material handling equipment. On the contrary, we do not consider the core process in the manufacturing system, which we believe is very different from product to product. The core process is also in most cases thoroughly analyzed in each company, whereas the material handling equipment is not.

\subsection{Autonomous}

By autonomous we mean that each material handling module has its own functionality, which is independent of all other modules: i.e., the smallest system possible consists of only one module.

\subsection{Modular}

The module itself has a specified functionality, which can be altered through the PLC, which has preprogrammed alternatives to choose from. The module boundaries are standardized and specified for safety, signals, electricity, and the carried loads are specified in three dimensions. The module itself has some degrees of freedom when it is created. Take for example one straight conveyor:
- Length
- Height
- Capacity
- Speed
- Width
- Direction

Figure 1 below shows one example when using a straight conveyor module. Other modules such as curves, incline/decline, buffers, transfers, turntables, manual workstations etc. do have other characteristics relative to their degrees of freedom.

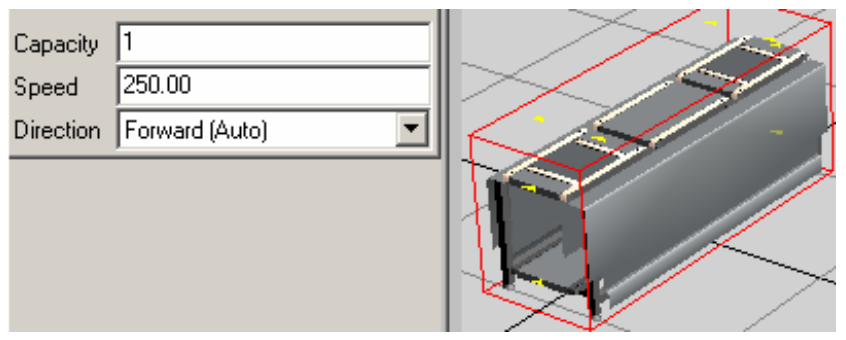

Figure 1: Straight Conveyor Module with Three Degrees of Freedom Visible

\subsection{Controlling the Modules}

Many of the controlling activities concerning a module are by nature local, i.e. they concern only the module itself and cooperating equipment such as neighboring modules. An example is the handshaking between two connected conveyors. Control code for such activities is therefore easy to reuse and often beneficial to distribute. Activities such as routing of products might have global impact on balancing and blocking situations. A central controller is therefore still recommended, in addition to the distributed module controllers.

\subsection{Module Behavior}

Along with data concerning properties such as width, height, and capabilities, the module might also exhibit data that describes its behavior. For control purposes, the behavior described by a discrete event model is of particular interest. By modeling the possible and desired behavior of the module in a formal manner, this model can be used for generating the control code for the module itself, and also function as input to simulations and formal verifications of the manufacturing system. Modular process models result in high degree of flexibility in reconfiguration of the system and in introduction of new products (Adlemo et al. 1995, Fabian et al. 1997). Code generation would guarantee that the model coincides with the actual behavior.

\subsection{Global Behavior}

If each module knows the identity of its connected neighbors, and this information can be accessed, then it would be easy to get a global view of how the modules are arranged. The identification is preferably done automatically upon connection. If the discrete event model of each module can be extracted, and the arrangement of the modules is known, then we can construct a global model of the system. This model describes the possible behavior of the 
system. The risk of inducing unwanted situations such as blocking increases as the manufacturing system becomes more complex. A global perspective is generally needed for discovering and avoiding such situations, which often can be considered as resource booking problems (Lennartson et al. 1998, Lennartson et al. 2002) By specifying what we allow or not allow the system to do, we can automatically generate a supervisor, using Supervisory Control Theory (Ramadge and Wonham 1987), that gives as much flexibility to the system as possible while avoiding blocked states and forbidden configurations.

\subsection{Routing}

In a flexible manufacturing system there can be several alternative routes for a product. The routing questions for a material handling module can be:

- From where to order? The question arises, if the system is using the pull approach, in modules where product flows merge.

- Where to send? Arises, if the system is driven by push, in modules that can split a product flow.

Answering those questions can be undertaken in different ways:

- Fixed routing. Each product has only one possible route. Probably the most common situation today.

- Static routing. The first currently feasible route in a priority list is selected.

- Local dynamic routing. The decision is based on some information of the current situation that the module has. This information can be based on queries to connected modules of the type: When can you deliver product A? How fast can you ship product $\mathrm{B}$ to the end station?

- Global routing. The central controller has most information on the current global situation of the manufacturing system, and should therefore be able to make optimal routing decisions.

\subsection{Virtual Tracking of Goods}

Keeping track of goods during manufacturing can be done in several ways, for example by using escort memory or bar codes. An alternative is to have virtual tracking of goods, where the control system tracks the order of the products without being able to identify the product by its escort memory or bar code. The concept with autonomous modules simplifies the implementation of virtual tracking, where each module is responsible for monitoring the products that it is carrying at the moment. The necessary product information is handed over to the next module simultaneously with the product itself.

\subsection{Virtual Development}

By drawing a clear distinction between the process model, i.e. the possible behavior of the system, and the controller, the control code developed for the simulation can also be used for controlling the real system. All information needed for programming the controller does already exist within the simulation. There should be no need for doing the same thing twice.

\subsection{Reconfigure the System}

The system can easily be reconfigured to handle other products and/or rearrange the routing of the products and the geometrical translations/rotations of the products both in the simulation model and in reality. A smaller rearrangement of a module or two including ramp-up of production takes less than five minutes for two persons to execute.

\section{SIMULATION SUPPORT}

The Discrete Event Simulation (DES) support tool used in this paper is the software package 3DCreate, and product derivatives 3DRealize and 3DVideo from Visual Components. This simulation package has proven to be the most suitable for line builders and machine providers in terms of modularization capabilities, learning curve, and the graphical representation of the software and its content (Johansson et al. 2004).

This kind of DES support has large benefits when it comes to lead time reduction in many aspects during the lifecycles of both products and production systems. A study regarding input data for DES shows that only $6 \%$ of industrial companies do have all required data for a DES model available (Johansson et al. 2003), which a modular DES approach would increase through reusability of input data as well as simplifications of the verification and validation steps of a simulation study (Banks et al. 2001).

As described in Johansson et al. (2004) simulation support for autonomous modular material handling equipment benefits form the following characteristics of the software:

- Modular library of "masters," i.e. offline representations of each standard module including its specifications and behaviour

- Pre-made logic built into each of these modules

- Degrees of freedom in each module, according to what is available in the real word, i.e. length of conveyors, width of conveyors, buffer size etc.

- Real scale 3-D graphical representation of each module

- Easily manageable connection points for each module, including connection type, i.e. logics, material, operator etc. 
However, the software is not simplified in all aspects, as will be clarified in the next section.

\section{USER KNOWLEDGE LEVEL DEMANDS}

To be able to handle all aspects of this simulation approach a person needs to be as skilled with this software as with any other software such as, for example, QUEST, Automod or WITNESS. However, with a modular approach only one or two people in larger companies need this competence level and will develop the modules, while all the other employees and sales personnel need only have the basic simulation skills to build a model out of the predefined modules. Even less skill is required to run a simulation model.

According to the models used for vocational knowledge and learning described in Nordell et al. (2003), this modular simulation concept will package some of the simulation expert's explicit and tacit knowledge into the software module representation of the real module, which will be used only by the simulation user without any demands on understanding the internal structure of the module. In other words, the simulation expert's knowledge will be embedded into the module, which simplifies and lessens the demands on knowledge for the simulation user. Traditionally a continuous striving towards converting tacit knowledge to explicit knowledge is desired. However, this is not an easy task, since tacit knowledge is "Unutterable and Unarticulated." But the modular approach of simulating autonomous modular material handling equipment simplifies the conversion to a large extent. Figure 2 below shows the relations of tacit and explicit knowledge, modified from Nonaka (1994) and Gustafsson (1999).

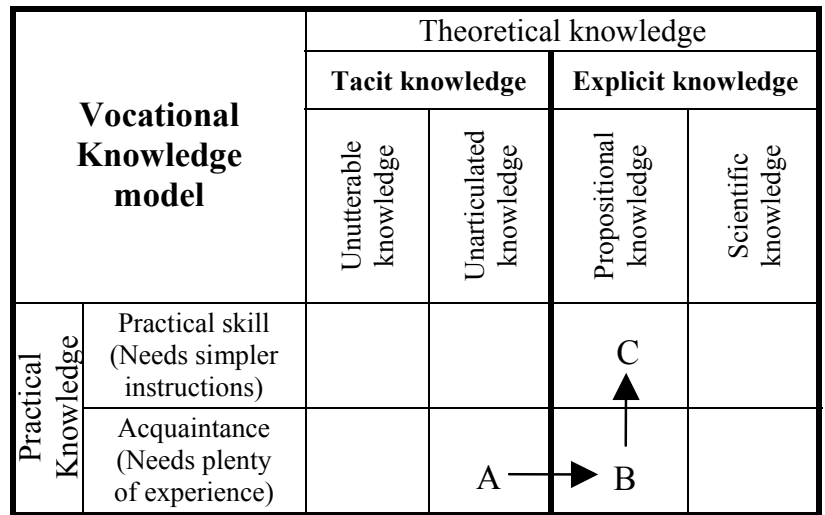

Figure 2: Matrix Model of the Different Aspects of Vocational Knowledge

This figure can be used to explain how the knowledge is transformed from tacit to explicit. When the simulation expert is formulating the modules from his mind into the computer (from A to B), the knowledge will change form from tacit to explicit on the acquaintance level. Then the simulation software package architecture does the rest of the work while using the modular approach by transforming the ex- plicit knowledge from acquaintance to practical skill (from B to C), which can then be used by the simulation users with less experience. Traditional simulation software packages does only make the transformation from $\mathrm{A}$ to $\mathrm{B}$, which then sets the simulation user in the acquaintance level of propositional knowledge. This level requires many months of experience to master when it comes to traditional simulation software packages. However, the traditional packages can be used with tailor-made user interfaces to enable the same kind of modularity as in the Visualcomponents software package.

In the coming three subsections a description of skills and their knowledge level needed for each type of user in the modular approach is described.

\subsection{Simulation Specialist}

In order to build modules out of nothing, the specialist skills are at about the same level as before, such as when using traditional simulation software, Extend, WITNESS, QUEST, Automod, ED, etc...

Needed skills for the simulation specialist are:

- Advanced Computing

- Advanced Programming

- General CAD

- Advanced mathematical skills in terms of statistics and probability.

Lead time for skill development for a non simulation specialist is long, approximately half a year. Typical users are module designer and simulation specialists who belong to "A" in the Vocational knowledge model.

\subsection{Simulation User}

In order to build simulation models out of the predefined modules made by a specialist, the simulation user needs awareness of the system impacts form the different modules, as well as general system knowledge, in this case manufacturing systems knowledge.

Needed skills for the simulation user are:

- General computing

- General production system

- General statistics.

Lead time for skill development for a non simulation user is short, approximately a few days. Typical users are sales personnel, system builders, plant designers, system integrators, continuous improvement personnel who belong to " $\mathrm{C}$ " in the vocational knowledge model.

\subsection{Simulation Observer}

In order to watch and run the simulation models made by the simulation user out of the simulation expert made mod- 
ules, the simulation observer does not need to have any skills beforehand at all, except for being able to handle a computer for normal work activities.

Needed skills for the simulation observer:

- General computing.

Lead time for skill development for a non simulation observer is very short, approximately five minutes. Typical users are everybody with interest of the system, but especially managers and operators, who belong to " $\mathrm{C}$ " in the vocational knowledge model.

\section{LEAD TIME REDUCTION}

Discrete Event Simulation as a lead time reducer is the main contributor for productivity improvements for both the line builder and the user of the autonomous modular material handling equipment. Lead time reduction can be identified in several stages during the lifecycle of both the products and the autonomous modular material handling equipment.

\subsection{Sales Process}

Early offering stages when no real system exists, the customer and the line builder salesman can share, build, and discuss different layouts and concepts for the manufacturing system in order to prevent expensive mistakes.

\subsection{Manufacturing Line Design Process}

Predefined modules give the possibility for automatic generation of $\mathrm{BOM}$, parts list, drawings etc. This integration will dramatically decrease the effort for CAD drawings and rework of additional BOM's and part lists for each and every project.

\subsection{Implementation Process}

The real-world implementation process will be shorter and more accurate since more testing and validation can be made offline and offsite (It can be done at the line builder and not necessarily at the customer.)

\subsection{Operational Process}

During the operational phase of the autonomous modular material handling equipment, the simulation model of the system can be used for production planning and testing of future production, as well as be connected to the daily work in the system, such as surveillance, maintenance, and continuous improvements activities.

\subsection{Reconfiguration Process}

Large lead time reductions can also be attained when NPI (New Product Introduction) is going to take place in the manufacturing system. The DES model can then be used for testing various possible scenarios for reconfiguration of the system, product mix and batching, additional capacity requirements etc. Since the simulation model already exists, only minor changes in layout and products will be needed in order to find a new solution for future manufacturing and reusability of the modules for the next generation of products.

\subsection{Other Processes}

Internally at the line building company, other processes can also be reduced in their effort and lead time. New modules can be created and tested offline by internal technicians, in the virtual environment before the construction of real modules in sales situations, thereby shortening and enhancing the introduction of the new module into the presently available modules.

\section{DISCUSSION}

As always when it comes to implementing new technology into an existing organisation, there will be barriers and hindrances to overcome in order to be successful. According to Östman (1998), the technological challenge is only a minor part when it comes to implementing new technologies, compared to development of working procedures and integration of them into the existing organisation.

Another requirement on the organisational aspects is competence development and learning activities for all employees in order to face the implementation and use of the new technology. The learning activities needs to be accompanied with information and plans on how the development evolves, and what is to be expected in the near future. Such activities will enable all employees to become part of the implementation and the success rate will increase.

Even though the presented approach is striving towards standardisation, the real world today always requires some special solution in the implementation phases. However, if $80 \%$ of the solution can be made with a standardized approach, there is much to be gained. Compare that with today's activities where most solutions are made as a one-offsolution with no possibilities for reuse and reconfiguration.

\subsection{Module Leasing Activities}

By using this approach the reusability of the equipment, data and processes is then very high. A real proof that the equipment is fully modularized appears when it can be offered as short term leasing or renting modules by suppliers. The investment need with this approach is far lower than traditional "one off kind solutions", and also spread over time. The user invests only when a capacity need arises and de-invests when it's the opposite, tightly connected to marketing demands or order intake. SME's, which are struggling with losing jobs to low-cost countries, have now a 
way of automating with a high flexibility at a low investment level. At the ISR2002 in Stockholm the PLM Factory concept proved the above is reality today (Bagiu and Johansson 2004).

\subsection{Future Module Compatibility}

An issue which needs further attention is version handling, which needs to be addressed in every PDM system. This issue is not yet solved for autonomous modular material handling equipment. However, since almost all PDM systems have that feature, it should be a minor issue to solve it for this technology. But to enable old and new modules to be compatible with each other in an integrated system -that is more of a challenge.

\section{CONCLUSION}

In this paper we have presented how support from a modular DES software can generate benefits and lead-time reductions in all lifecycle stages of products and manufacturing processes for autonomous modular material handling equipment. The conclusion indicates that the benefits from using DES are numerous and the potential is obvious. The effort of implementing these technologies is another step towards making simulation a corporate norm (Williams 1996).

\section{FUTURE RESEARCH}

The approach described in this paper coincides with development and implementation at FlexLink in Sweden and will be further developed during the years to come, however additional research is required in many fields in order to complete the full concept. Areas where efforts are needed are for example: Standards, version handling of new and old equipment, safety and regulation adaptations, and holistic systems control functions. However, small steps, one at a time, will bear fruit each year in quest for the optimal solution for autonomous modular material handling equipment for manufacturing flexibility.

\section{ACKNOWLEDGMENTS}

The funding for this research is granted by FlexLink AB, IMIT (Institute for Management of Innovation and Technology), VINNOVA (Swedish Agency for Innovation Systems, integrates research and development in technology, transport and working life.) and Wingquist Laboratory.

\section{REFERENCES}

Adlemo, A., S.-A. Andreasson, M. Fabian, P. Gullander, and B. Lennartson. 1995. Towards a Truly Flexible Manufacturing System, Control Engineering Practice 3, (4): 545-554.
Bagiu, J., and B. Johansson. 2004. Profitable Intelligent Manufacturing Systems for the Future, In Proceedings of the $35^{\text {th }}$ International Symposium on Robotics, SYMAP, 23-26 March, Paris-Nord Villepinte, France.

Banks, J., J.S. Carson, B.L. Nelson, and D. Nicol. 2001. Discrete Event System Simulation, $3^{\text {rd }}$ ed., Upper Saddle River, New Jersey: Prentice-Hall.

Fabian, M., B. Lennartson, P. Gullander, S.-A. Andreasson, and A. Adlemo. 1997. Integrating Process Planning and Control for Flexible Production Systems, In Proceedings of the 4th European Control Conference, 1-4 July, FR-M K1. Brussels, Belgium: European Union Control Association.

Gustafsson, B. 1999. On the Importance of Integrating Hand and Mind in the Automation Process, Licentiate Thesis (In Swedish), Department of Product and Production Development, Chalmers University of Technology, Gothenburg, Sweden, ISSN 1100-7524, PTA: 99:05.

Johansson, B., J. Johnson, and J. Bagiu. 2004. Modular Assembly Systems Simulation for Lead Time Reduction, In Proceedings of the International Precision Assembly Seminar, ed Ratchev S., Delchambre, A., 215-222, Bad Hofgastein, Austria.

Johansson, B., J. Johnsson, and A. Kinnander. 2003. "Information Structure to Support Discrete Event Simulation in Manufacturing Systems". , Proceedings of the 2003 Winter Simulation Conference, eds S. Chick, P. J. Sánchez, D. Ferrin, and D. J. Morrice, 1290-1295, Dec. 7-10, New Orleans, U.S.A. IEEE, Piscataway, NJ.

Koren, Y., U. Heisel, F. Jovane, T. Moriwaki, G. Pritchow, H. Van Brussel, and A.G. Ulsoy. 1999. Reconfigurable Manufacturing Systems, CIRP Annals 48, (2): 527-540.

Koren, Y., A.G. Ulsoy, R.G. Landers, M. Mehrabi, A. Pasek, and D. Yip-Hoi. 2001. NSF Engineering Research Center for Reconfigurable Machining Systems, In Proceedings 2001 NSF Design, Manufacturing and Industrial Innovation Research Conference. Jan 7-10, 2001, Tampa, Florida, U.S.A.

Lennartsson, B., M. Fabian, and P. Gullander. 2002. CHAMP - a Generic Architecture for Flexible Production, Proceedings of the $33^{\text {rd }}$ International Symposium on Robotics, paper 022. Stockholm, Sweden: International Federation of Robotics.

Lennartson, B., M. Fabian, M. Tittus, and A. Hellgren. 1998. Modeling primitives for supervisory control, Proceedings of WODES '98, 67-72. Cagliari, Italy: Institution of Electrical Engineers.

Mehrabi, M.G., and A.G. Ulsoy. 1997. State-of-the-Art in Reconfigurable Manufacturing Systems, Internal Report, ERC/RMS. Report No. 2, Vol. 1. Department of Mechanical Engineering and Applied Mechanics, University of Michigan, Ann Arbor.

Nahmias, S. 2001. Production and Operations Analysis, $4^{\text {th }}$ edition. Boston, Massachusetts: The McGraw-Hill Companies, Incorporated. 
Nonaka, I. 1994. A Dynamic Theory of Organizational Knowledge Creation, in Organization Science 5, (1), February.

Nordell, P., B. Gustafsson, and A. Kinnander. 2003. Knowledge-Based Method for Efficient Reduction of Losses in Manufacturing Lines, The $36^{\text {th }}$ CIRP-International Seminar on Manufacturing Systems, June 03-05, Saarland University, Saarbruecken, Germany.

Östman, J. 1998. Virtual Reality och Virtual Prototyping Tillämpningar i Amerikansk Fordonsindustri (In Swedish), Utlandsrapport USA 9806, Swedish Office of Science and Technology, Sweden.

Ramadge, P., and W. Wonham. 1987. Supervisory Control of a Class of Discrete Event Processes, SIAM J. Control Optim. 25, (1): 206-230.

Wickens, C. D., D. L. John, L. Yili, and E. G. B. Sallie. 2004. An Introduction to Human Factors Engineering, $2^{\text {nd }}$ edition. Upper Saddle River, New Jersey: Pearson Education, Incorporated.

Williams, E. J. 1996. Making Simulation a Corporate Norm. In Proceedings of the 1996 Summer Computer Simulation Conference, eds. V. Wayne Ingalls, Joseph Cynamon, and Annie Saylor, 627-632. San Diego, Society for Computer Simulation International.

\section{AUTHOR BIOGRAPHIES}

BJÖRN JOHANSSON was born in Gothenburg, Sweden, 1975. He attended Chalmers University of Technology at Mechanical Engineering, where he obtained his M.S. degree in Production Engineering in 2000, and his Licentiate Degree in 2002. He is now working as a PhD student in the field of Discrete Event Simulation and Productivity Improvements in Manufacturing Systems at the Department of Product and Production Development, Chalmers University of Technology, Sweden. His email address is <Bjorn. Johansson@me. chalmers.se>.

EDWARD J. WILLIAMS holds bachelor's and master's degrees in mathematics (Michigan State University, 1967; University of Wisconsin, 1968). From 1969 to 1971, he did statistical programming and analysis of biomedical data at Walter Reed Army Hospital, Washington, D.C. He joined Ford Motor Company in 1972, where he worked until retirement in December 2001 as a computer software analyst supporting statistical and simulation software. After retirement from Ford, he joined Production Modeling Corporation, Dearborn, Michigan, as a senior simulation analyst. Also, since 1980, he has taught evening classes at the University of Michigan, including both undergraduate and graduate simulation classes using GPSS/H, SLAM II, SIMAN, ProModel, SIMUL8, or Arena ${ }^{\circledR}$. He is a member of the Institute of Industrial Engineers [IIE], the Society for Computer Simulation International [SCS], and the Michigan Simulation Users' Group [MSUG]. He serves on the editorial board of the International Journal of Industrial Engineering - Applications and Practice. During the last several years, he has given invited plenary addresses on simulation and statistics at conferences in Monterrey, México; Istanbul, Turkey; Genova, Italy; and Riga, Latvia. His e-mail address is: <williame@umdsun2. umd. umich.edu> and his university Web-page can be found at <www. personal. umd. umich. edu/ williame. coeditor $>$ for the WSC 2004 conference.

TORD ALENLJUNG was born in Mariestad, Sweden, 1977. He obtained a master's degree in Automation and Mechatronics at Chalmers University of Technology in 2001. During 2001-2003 he worked as a software developer at Carmenta, Gothenburg, developing, among other things, an air traffic surveillance system. Since 2003 he is working as a PhD student at the Department of Signals and Systems, Chalmers University of Technology, Sweden, where his field of research is in specification and modeling of discrete event systems. His email address is <tordae s2. chalmers.se>. 